\title{
Utility of magnetic source imaging in nonlesional focal epilepsy: a prospective study
}

\author{
Ismail Sidky Mohamed, MD, FRCPC, ${ }^{4,5}$ Dènahin Hinnoutondji Toffa, MD, PhD, CSCN, ${ }^{1}$ \\ Manon Robert, MSc, TEPM, ${ }^{3}$ Patrick Cossette, MD, PhD, FRCPC, ${ }^{1}$ \\ Arline-Aude Bérubé, MD, FRCPC, ${ }^{1}$ Jean-Marc Saint-Hilaire, MD, FRCPC, ${ }^{1}$ \\ Alain Bouthillier, MD, FRCSC, ${ }^{2}$ and Dang Khoa Nguyen, MD, PhD, FRCPC1 \\ Divisions of ${ }^{1}$ Neurology and ${ }^{2}$ Neurosurgery, Montreal University Health Center, Université de Montréal, and ${ }^{3}$ Neuropsychology \\ and Cognition Research Center, Psychology Department, Université de Montréal, Quebec, Canada; and Departments of \\ ${ }^{4}$ Pediatrics, Division of Neurology, and ${ }^{5}$ Neurology, University of Alabama, Birmingham, Alabama
}

\begin{abstract}
OBJECTIVE For patients with nonlesional refractory focal epilepsy (NLRFE), localization of the epileptogenic zone may be more arduous than for other types of epilepsy and frequently requires information from multiple noninvasive presurgical modalities and intracranial EEG (icEEG). In this prospective, blinded study, the authors assessed the clinical added value of magnetic source imaging (MSI) in the presurgical evaluation of patients with NLRFE.
\end{abstract}

METHODS This study prospectively included 57 consecutive patients with NLRFE who were considered for epilepsy surgery. All patients underwent noninvasive presurgical evaluation and then MSI. To determine the surgical plan, discussion of the results of the presurgical evaluation was first undertaken while discussion participants were blinded to the MSI results. MSI results were then presented. MSI influence on the initial management plan was assessed.

RESULTS MSI results influenced patient management in 32 patients. MSI results led to the following changes in surgical strategy in 14 patients (25\%): allowing direct surgery in 6 patients through facilitating the detection of subtle cortical dysplasia in 4 patients and providing additional concordant diagnostic information to other presurgical workup in another 2 patients; rejection of surgery in 3 patients originally deemed surgical candidates; change of plan from direct surgery to icEEG in 2 patients; and allowing icEEG in 3 patients deemed not surgical candidates. MSI results led to changed electrode locations and contact numbers in another 18 patients. Epilepsy surgery was performed in 26 patients influenced by MSI results and good surgical outcome was achieved in 21 patients.

CONCLUSIONS This prospective, blinded study showed that information provided by MSI allows more informed icEEG planning and surgical outcome in a significant percentage of patients with NLRFE and should be included in the presurgical workup in those patients.

https://thejns.org/doi/abs/10.3171/2020.1.FOCUS19877

KEYWORDS magnetoencephalography; prospective study; nonlesional; epilepsy surgery

$\mathrm{E}$ PILEPSY surgery can be curative in patients with medication-resistant epilepsy and seizures localized to an area of the brain where surgical resection can be safely performed. ${ }^{27}$ The presence of a structural epileptogenic lesion on brain MRI significantly improves surgical outcome, as the location of the lesion is usually concordant with the epileptogenic zone (EZ). ${ }^{22}$ However, approximately $25 \%$ of medication-resistant focal epilepsies are considered nonlesional. ${ }^{2}$ In patients with nonle- sional refractory focal epilepsy (NLRFE), presurgical mapping of the EZ will depend on data from other noninvasive modalities, including scalp video-EEG (VEEG), ictal and interictal SPECT, and PET. ${ }^{19}$

Magnetoencephalography (MEG) measures extracranial magnetic fields generated by neuronal currents and is being increasingly used in the presurgical evaluation of medication-resistant epilepsy. ${ }^{7,9,14-16,26}$ In clinical practice, MEG source localization is typically performed us-

ABBREVIATIONS ATL = anterior temporal lobectomy; ECD = equivalent current dipole; EMSI = electromagnetic source imaging; $E Z$ = epileptogenic zone; icEEG = intracranial EEG; MEG = magnetoencephalography; MSI = magnetic source imaging; NLRFE = nonlesional refractory focal epilepsy; SEEG = stereo-electroencephalography; VEEG = video-EEG

SUBMITTED November 11, 2019. ACCEPTED January 28, 2020.

INCLUDE WHEN CITING DOI: 10.3171/2020.1.FOCUS19877. 
ing equivalent current dipole (ECD) modeling of interictal spikes. The location and orientation of the estimated spike sources are then coregistered and overlaid onto the patient's MRI to create magnetic source images. The magnetic source imaging (MSI) results are then used in combination with data from other noninvasive modalities to guide surgical decisions. Previous studies have shown that MSI can provide clinically pertinent and complementary information about the location and extent of the EZ in about $20 \%-30 \%$ of patients with refractory focal epilepsy. MSI results have been used to guide patient selection for surgery, have improved the accuracy of invasive EEG electrode placement, and may lead to improved surgical outcome..$^{1,3,11,13,20}$ Despite numerous studies showing its utility, MSI use is still limited to a few centers across the world. The lack of widespread use of MSI across centers can be attributed to the high prices of MEG systems and their operating costs, and also to the relative lack of wide-scale prospective studies assessing its usefulness. Many studies examining the utility of MSI have been limited by small sample size, indiscriminate inclusion of both lesional and nonlesional cases, relatively short-term seizure outcomes, or a combination of these factors.

We prospectively examined the impact of MSI on surgical decision-making in a cohort of patients with NLRFE.

\section{Patients and Methods \\ Study Context}

An MEG system was installed at our university in 2006 by a group of cognition researchers. ${ }^{14}$ Benefiting from the availability of MEG, we designed a research project to evaluate the utility of MEG for patients with NLRFE. The study was approved by our institutional ethics committee and all patients provided signed consent forms. From April 2006 to January 2010, MSI results were not included in preoperative surgical decision-making as the acquired MEG data could not be analyzed in a timely fashion, different source analysis methods were being explored, and MEG results were still felt to be experimental. The impact of the lack of MEG utilization in this group has been previously published. ${ }^{14}$ For subsequent patients, MSI results were presented during the epilepsy surgery case management conference after review of standard presurgical workup.

\section{Patients}

During January 2010 through October 2017, all patients with NLRFE undergoing evaluation for potential epilepsy surgery were recruited. Patients with nonspecific changes in MRI studies (e.g., small white matter changes, mild diffuse atrophy) or with a lesion probably unrelated to the epileptic condition (e.g., Chiari malformation, arachnoid cyst) and patients with prior nonlesional epilepsy surgery failures who presented with postoperative changes on MRI were included. Patients with mesial temporal lobe epilepsy were excluded if imaging showed ipsilateral mesial temporal sclerosis on MRI. The standard presurgical evaluation included VEEG recording of seizures, high-resolution cerebral MRI (Achieva Dual 3.0T system, Philips Medical Systems), ictal SPECT, and ${ }^{18}$ fluoro-deoxy-glucose PET.

\section{Magnetic Source Imaging}

We obtained MEG recordings while patients were admitted for long-term VEEG monitoring as part of their presurgical evaluation. For most patients, antiepileptic drugs had frequently been tapered or dosages lowered and patients were in a state of mild sleep deprivation (4 hours) for the MEG study.

MEG recordings were obtained using a whole-head 275-sensor MEG system in a magnetically shielded room (CTF-MEG, Coquitlam). MEG and concomitant EEG data were acquired in multiple datasets, each 5 minutes in duration, for an average total duration of 90 minutes at a sampling rate of $600 \mathrm{~Hz}$. MEG data were analyzed by an epileptologist (I.S.M.) who was experienced in MEG interpretation and only had access to a sample of the morphology and localization of spikes recorded during scalp VEEG monitoring obtained during VEEG monitoring. The parameters and process of ECD analysis of MEG data were described previously. ${ }^{14}$

According to guidelines of the American Clinical MEG Society, reliable results require that sources for at least 5 epileptiform discharges be identified from a given patient. Based on these guidelines, MEG datasets were classified into 4 categories: ${ }^{8,9,16} 0=$ no MEG spike sources ( $<5$ MEG spike sources, which yields insignificant findings); $1=$ single cluster ( $\geq 6$ spike sources with $\leq 1 \mathrm{~cm}$ between each other); $2=$ multiple clusters; and $3=$ scatter (i.e., $\geq 6$ spike sources with $>1 \mathrm{~cm}$ between each other). Spike sources localized to the temporal lobe were defined as mesiobasal if located predominantly in the anterior half of the temporal lobe with horizontal or anterior vertical dipole orientation. Spike sources localized exclusively to the posterior half of the temporal lobe with vertical orientation were classified as localized to the lateral temporal lobe. ${ }^{5,12}$ MSI findings were defined as localized if only 1 cluster or 1 cluster plus scatter was detected. MSI was considered multifocal or inconclusive if more than 1 cluster or scatters only were found, or if no spikes were detected during the recording.

\section{Assessment of MSI Contribution to Patient Management}

Anonymized results of the standard presurgical evaluation for all patients were presented randomly to a multidisciplinary epilepsy surgery team (blinded to MSI results). This team included 3 epileptologists (A.A.B., P.C., and J.M.S.H.) who had not previously been involved in the care of the patients discussed. For each patient, the presumed localization of the EZ (temporal or extratemporal) and the plan of action (A, focal resective surgery without intracranial EEG [icEEG]; B, icEEG recordings; or C, rejection because patient was considered a poor surgical candidate unless new decisive information was obtained from MSI) were noted. When an icEEG was planned, the electrode locations were anatomically determined, and the total number of contacts was calculated. In a second step, MSI results were presented by a fourth epileptologist (D.K.N.) who had not taken part in the decision-making process. Any changes in the initial patient plan management or changes in initial icEEG planning to add or remove electrodes were noted. 


\section{Clinical Relevance of Changes in Patient Management Related to MSI Findings}

We determined the clinical relevance of MSI for the management of patients with a minimum of 18 months of follow-up. MSI clinical relevance was evaluated by assessing whether MSI findings led to any of the following clinical management changes: 1) inclusion of additional electrodes in the ictal onset zone or within the resection area; 2) reduction in the number of intracranial electrode contacts inserted; 3) change in the position of grid/strip electrodes; 4) motivation or demotivation to perform direct resection surgery instead of the initial plan; and 5) rejection of the surgical option.

\section{Correlation Between MSI and Surgical Outcome}

Surgical outcomes of patients for whom the resection included versus those for whom the resection did not include predominance of spike sources were compared using the Fisher's exact test. Engel class I and II patient outcomes were considered good, whereas Engel class III and IV outcomes were deemed poor. ${ }^{6}$

\section{Results \\ Study Population}

In total, 57 consecutive patients with NLRFE investigated for potential epilepsy surgery were included. Based on the standard presurgical evaluation, $32 / 57$ (56\%) were presumed to have nonlesional extratemporal lobe epilepsy and 25/57 (44\%) nonlesional temporal lobe epilepsy.

\section{MRI Findings}

MRI was normal in 47 patients. In the remaining 10 patients, MRI showed an arachnoid cyst in 3 patients, nonspecific white matter changes in 2 patients, and a nonspecific millimetric T2-signal abnormality in the subinsular region in 2 patients. In patients with a prior normal MRI, encephalomalacia related to a failed epilepsy surgery was found on a previous postoperative MRI in 2 patients and Chiari malformation type I in 1 patient. All of these lesions were considered to be nonlocalizing at the time of the evaluation.

\section{MSI Findings}

MEG captured spikes in 47 patients (82\%). Localized MSI results were found in 27 patients, including 1 cluster in 22 patients $(39 \%)$ and cluster plus scatter in 5 patients (9\%). The remaining 30 patients had multifocal or inconclusive MSI findings, including 10 patients who had no spikes detected.

\section{Influence of MSI Results on Patient Management}

MSI results led to changes in initial management in 32 patients (56\%, group A; Table 1). In 14 patients, MSI results led to the following changes affecting surgical strategy: allowing direct surgery in 6 patients by facilitating the detection of subtle cortical dysplasia in 4 patients (patients 5, 8, 9, and 31) and providing additional concordant diagnostic information used in other presurgical workups in 2 patients (patients 4 and 25); rejection of surgery in 3 patients (patients 6,24, and 28) originally deemed surgi- cal candidates; changing of the plan from direct surgery to icEEG in 2 patients (patients 10 and 20); and allowing icEEG in 3 patients (patients 18, 21, and 26) deemed not to be surgical candidates. In the remaining 18 patients, MSI results led to changes in electrode number or placement as follows: reducing the number of intracranial electrodes in 9 patients (patients 1, 2, 7, 11, 13, 17, 23, 27, and 30); changing subdural grid array position in 4 patients (patients 2 , 12,13 , and 16); and improving coverage through the placement of additional electrodes in 10 patients (patients 1-3, $14,15,19,22,27,29$, and 32), which resulted in increased numbers of contacts in 5 patients (patients 14, 15, 19, 22, and 29; Fig. 1).

\section{Clinical Relevance of MSI-Related Changes in Group A Patients}

MSI findings were instrumental in the decision-making in 12/32 (38\%) group A patients (12/57 [21\%] of the whole cohort), leading to the following changes: 1) insertion of additional depth electrodes and subdural contacts over the EZ in 5 patients (patients 1-3, 14, and 15), with all 5 patients having good surgical outcomes; 2) facilitation of the detection of subtle cortical malformations in 5 patients, allowing for direct surgery in 4 patients (patients 5, 8, 9, and 31) who underwent surgical resection with seizure-free outcomes, while the fifth patient (patient 6) elected not to have surgery because of overlap with the motor cortex; and 3) changing of the chosen intervention to icEEG in 2 patients. MSI findings allowed the detection of a left operculo-insular epileptogenic focus in patient 10 , originally planned for anterior temporal lobectomy (ATL). The patient became seizure free after left operculo-insular resection. One patient (patient 21) was deemed not to be a surgical candidate, but MSI showed a spike source cluster over the right precuneus leading to the decision to perform a right precuneus resection, after which the patient became seizure free.

In the remaining 20 patients, additional electrodes placed in 5 patients did not significantly affect outcome. Temporal depth electrodes were placed in patient 19, allowing capture of 1 hippocampal seizure; however, the left hippocampus was not removed, because the majority of seizures originated from the inferior rolandic region. Additional electrodes in patient 29 captured insular seizures in addition to mesial temporal seizures. Additional electrodes were also placed in patient 22 but did not affect the outcome as icEEG captured multifocal seizures. No seizures were captured from the added electrodes in 1 patient (patient 32), and no seizures at all were captured in 1 patient (patient 27); hence, the impact of additional electrodes cannot be estimated. No complications resulted from the additional electrodes in any of the 5 patients in whom the additional electrodes did not significantly affect outcome. However, because localized MSI findings eliminated the need for additional interhemispheric or orbitofrontal strips, MSI findings allowed reduction in the number of icEEG electrodes inserted but without clear impacts on outcome. Six of these patients had good surgical outcomes, and of the remaining 3, 1 patient (patient 23) had residual seizures as the resection was limited by overlap with the eloquent cortex. Another patient (patient 7) became free of habitual 


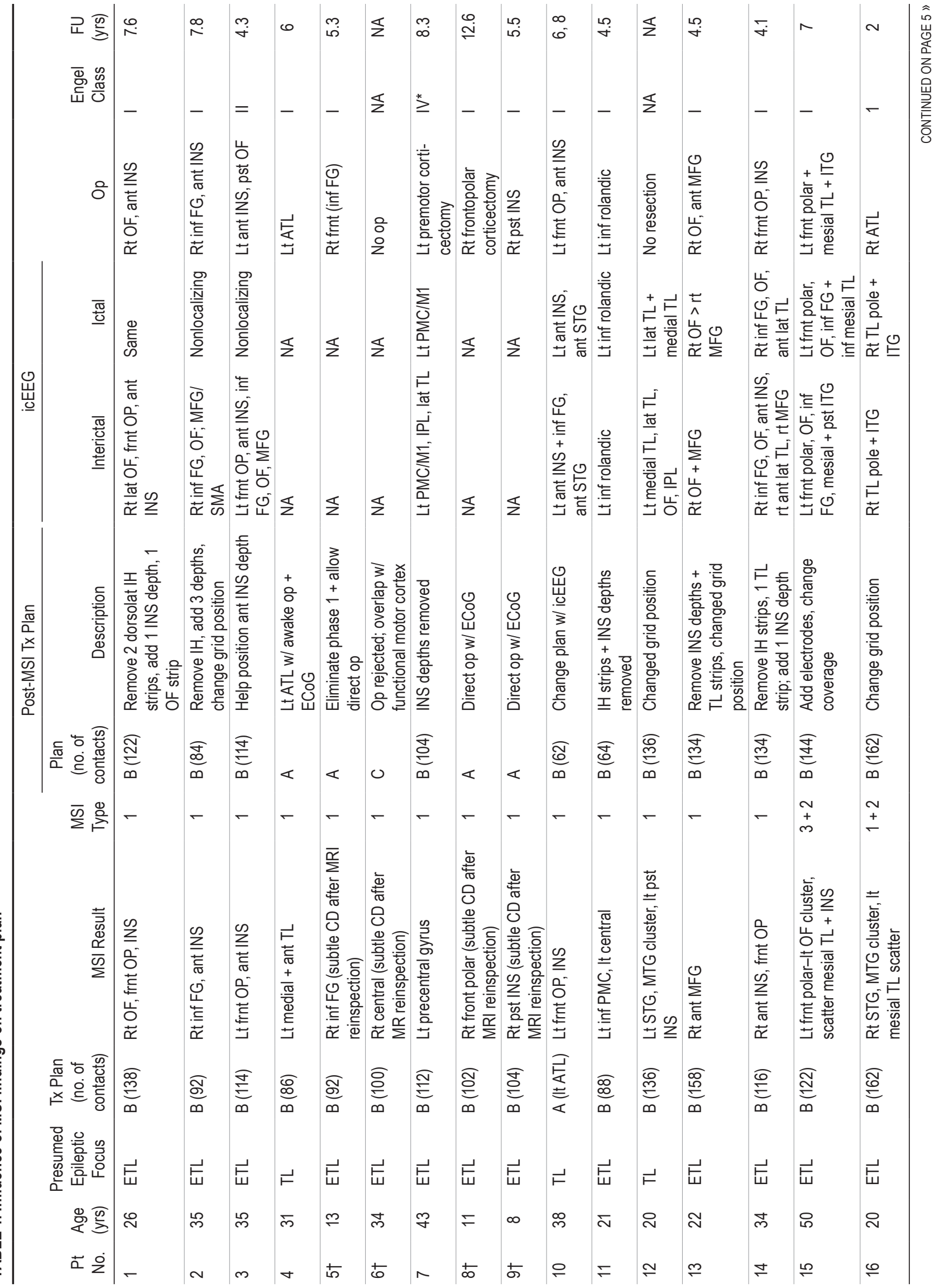




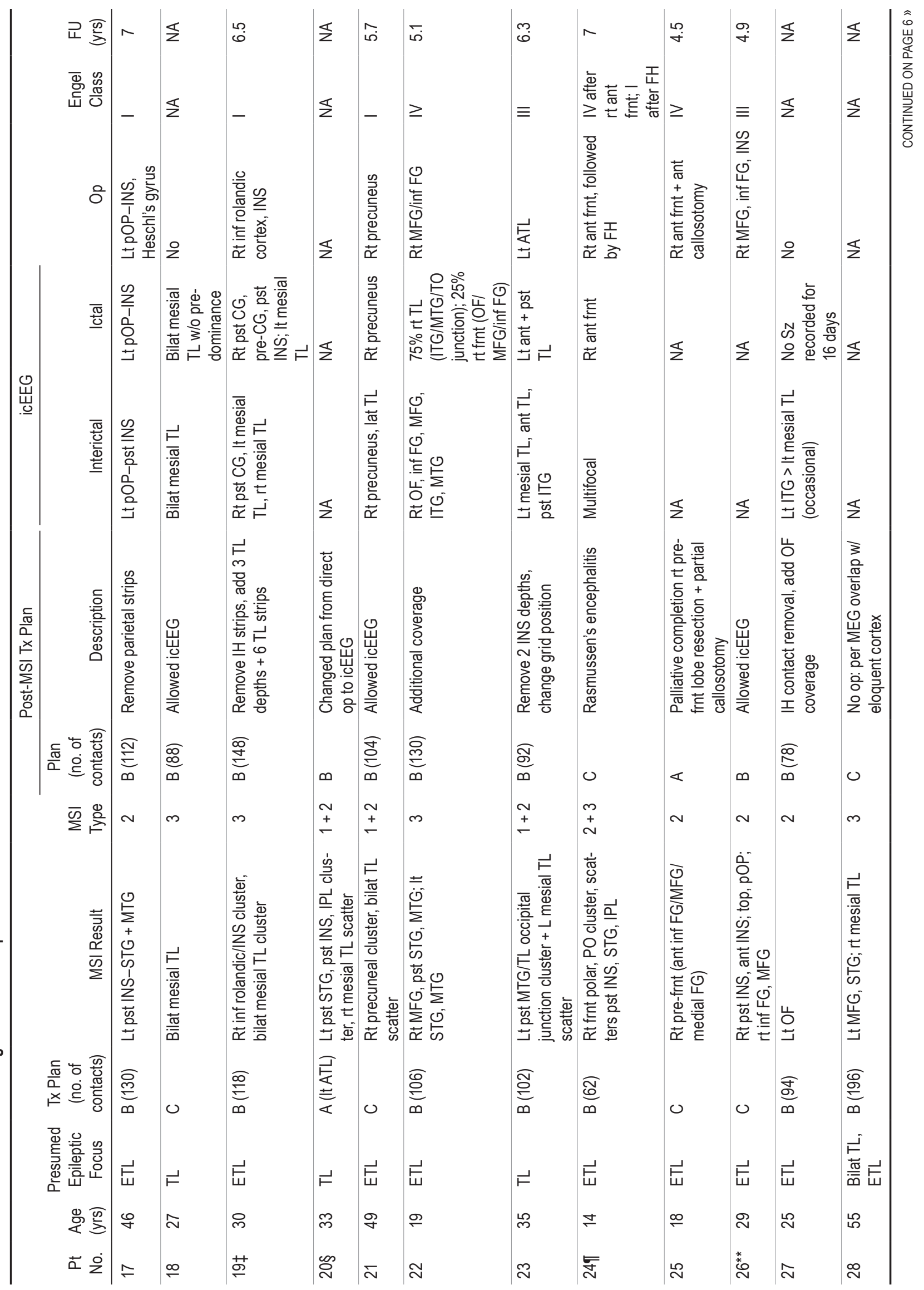




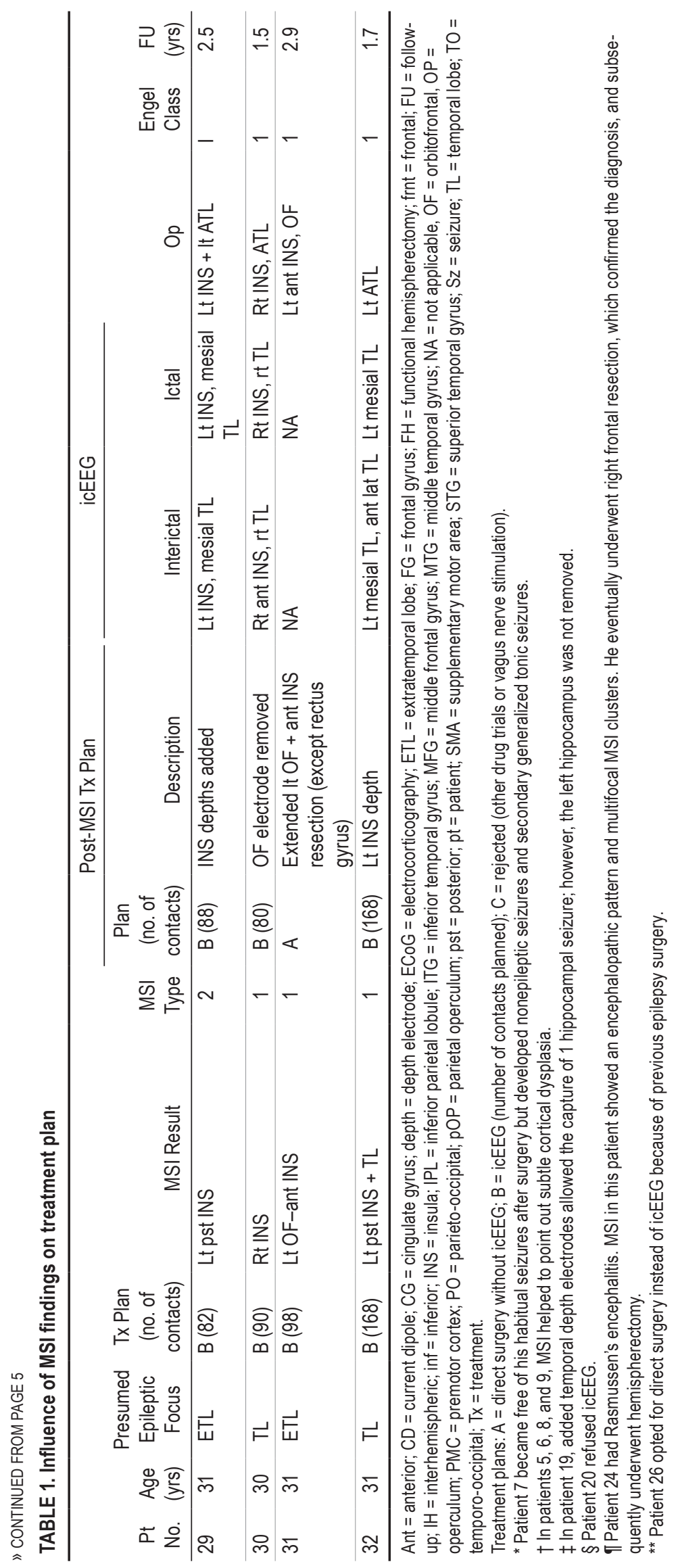




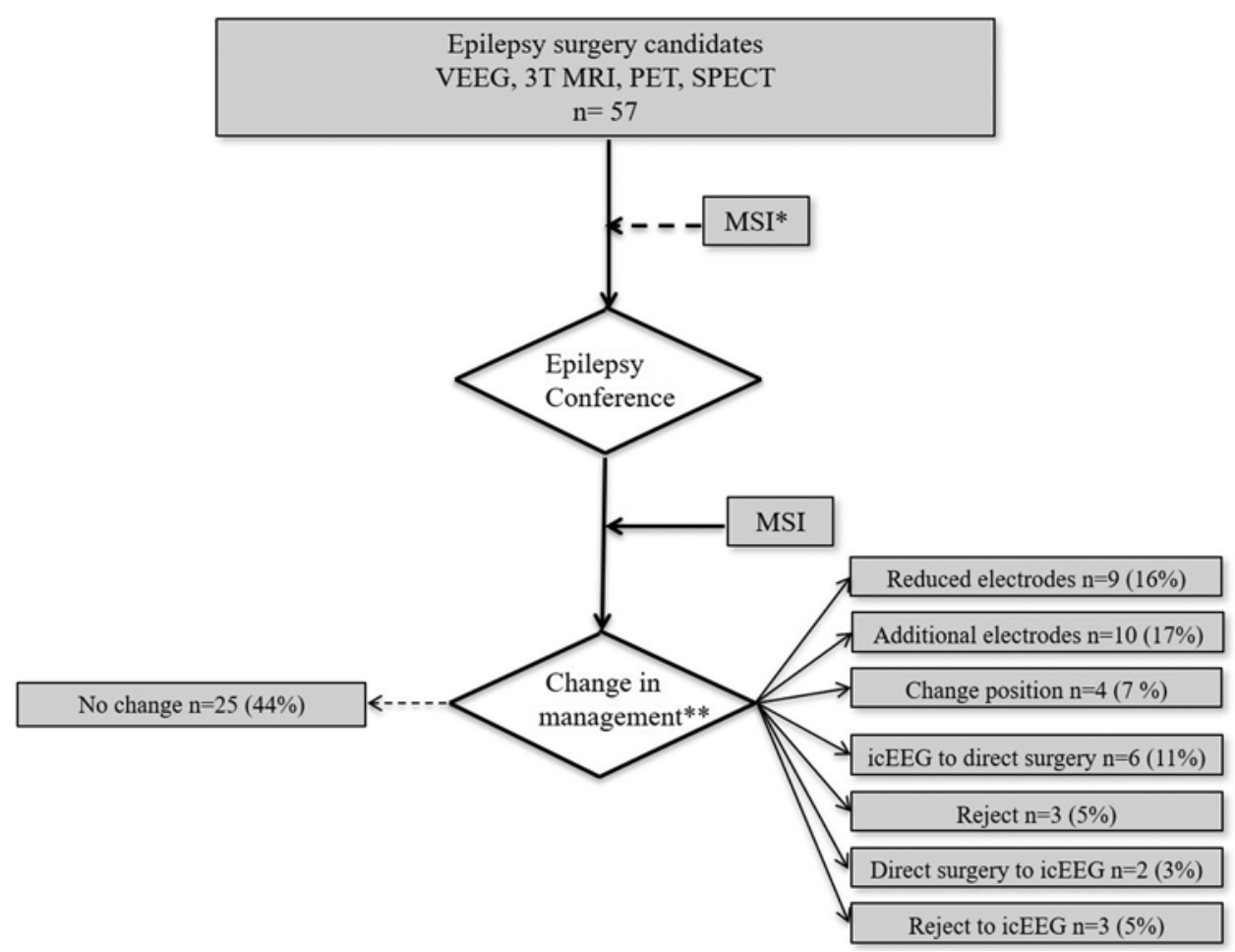

FIG. 1. Influence of MSI results on patient management. MSI findings led to changes in the initial treatment plan in $32 / 57(56 \%)$ patients. In 18 patients, MSI led to changes in electrode number or placement by reducing the number of intracranial electrodes in 9 patients, changing the subdural grid array position in 4 patients, and improving coverage through placement of additional electrodes in 10 patients. MSI results led to changes in surgical strategy in 14 patients by confirming direct surgery in 6 patients, the rejection of surgery in 3 patients originally deemed surgical candidates, changing the plan from direct surgery to icEEG in 2 patients, and confirming icEEG in 3 patients deemed not to be surgical candidates. ${ }^{*} \mathrm{MSI}$ results were not available at the time of the surgical decision. ${ }^{*}$ Change in patient management $(n=32,56 \%)$ as determined by consensus opinion after integration of MSI results.

epileptic seizures after surgery but developed nonepileptic seizures and secondary generalized tonic seizures. No seizures were captured in the third (patient 27) of the 9 patients during icEEG and no resection was performed. In 2 patients (patients 4 and 25), MSI information led to a change in the surgical planning decision by providing additional confirmatory information for other presurgical workup results. Patient 4 became seizure free after ATL. MEG likely did not affect the outcome since the original plan of icEEG covering the left temporal lobe would likely lead to left ATL. Surgery did not provide additional benefit in patient 25 , who underwent palliative completion of a previous right prefrontal resection and anterior partial callosotomy. Two patients were deemed not candidates for resective surgery after MSI results. In patient 28, MSI suggested multifocal epilepsy and potential overlap with the eloquent cortex. Patient 24 had Rasmussen's encephalitis. MSI in this patient showed an encephalopathic pattern with an abundance of high-voltage spike waves over the right hemisphere and multifocal MSI clusters. This patient eventually underwent right frontal resection, which confirmed the diagnosis, and subsequent hemispherectomy. In addition, MSI results led to changes in the subdural grid array position in 4 patients (patients $2,12,13$, and 16) to create a safety margin around the MEG cluster with no significant impact on outcome.

\section{Surgical Outcome}

Surgical resection was performed in 26 patients in group A. Good surgical outcome was achieved in 21 patients, while 5 patients had poor surgical outcome. Surgery included neocortical resections in 20 patients, ATL in 2 patients, and ATL plus (ATL combined with focal resection of the perisylvian cortex) in another 2 patients. Follow-up durations ranged from 18 months to 12.6 years (mean $5.97 \pm 1.32$ years). No resection was performed after icEEG in 3 patients due to overlap with eloquent cortex (patients 12 and 18) or no seizures captured during icEEG (patient 27) (Fig. 2). In group B, good surgical outcome was obtained in 13 patients and poor surgical outcome in 2 patients during follow-up that ranged from 19 months to 8.5 years (mean $5.53 \pm 1.30$ years). Surgery included neocortical resections in 8 patients, ATL in 6 patients, and ATL plus in 1 patient. No surgery was performed in the remaining 8 patients (Fig. 2). There was no significant statistical difference in outcome between the 2 groups.

\section{Correlation Between MSI, icEEG Findings, and Surgical Outcome}

Surgical resection was performed in 23 of $27(85 \%)$ patients with localized MSI findings. Surgical resections included ATL in 5 patients and neocortical resections in 

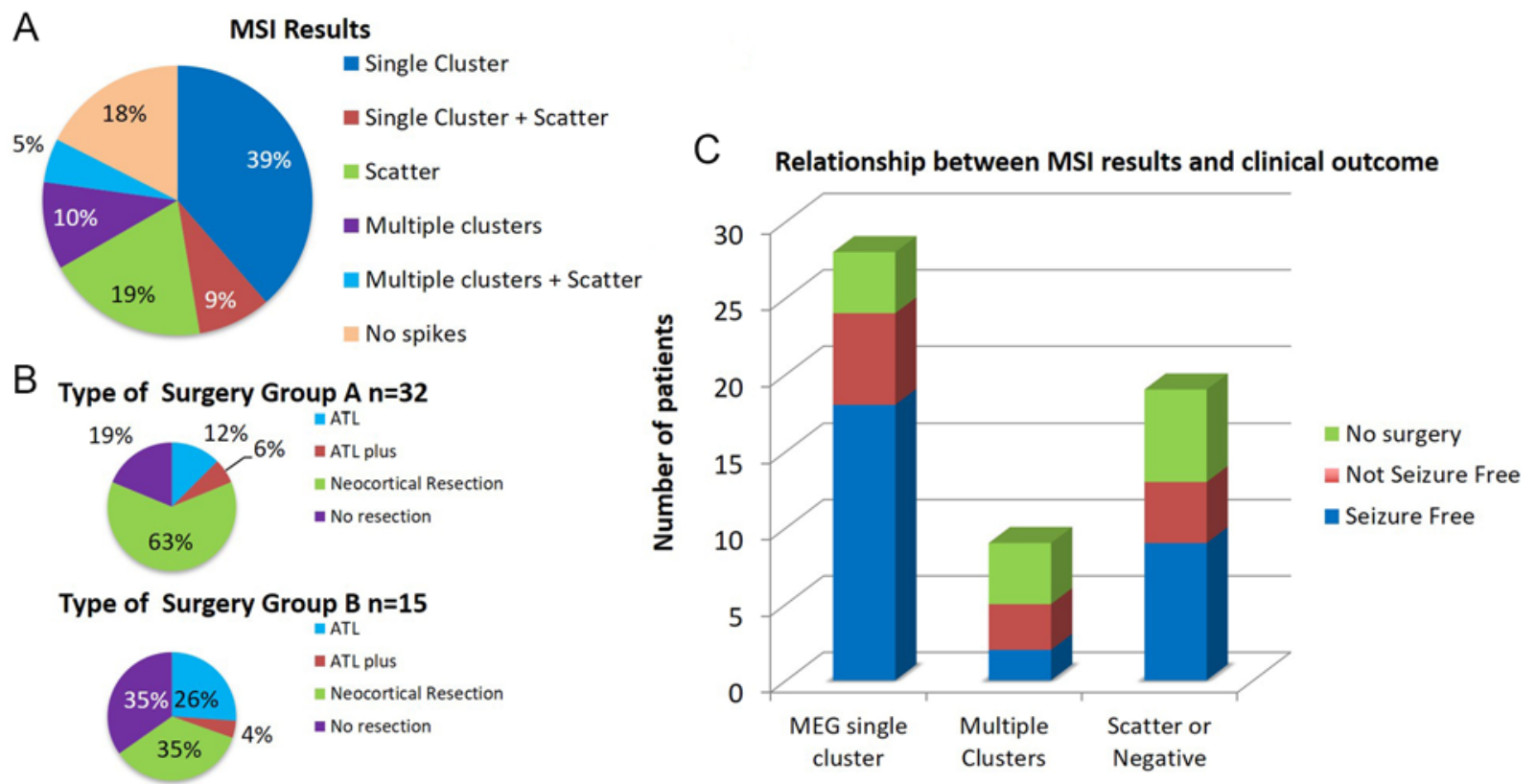

FIG. 2. MSI results and clinical correlation. A: Types of MSI result: a single cluster was the most frequent result. B: Types of surgery performed in the group of patients whose MSI results led to changes in the initial plan (group A) and in the other patients (group B). C: Relationship between MSI results and clinical outcome: a single cluster is more frequently associated with a good outcome than are multiple clusters.

the remaining 18 patients. Good surgical outcome was achieved in 19 patients (83\%), including 18 patients with Engel class I outcome.

Surgical resection was performed in 18 patients $(67 \%)$ with inconclusive or multifocal MSI findings. Surgical resections included ATL in 5 patients and neocortical resections in 13 patients. Good surgical outcome was achieved in 13 patients (72\%), including 10 patients with Engel class I outcome.

\section{Discussion}

This study provides further evidence for the role of MSI in the presurgical workup of patients with refractory nonlesional epilepsy. There are few previously reported studies that prospectively examined the role of MSI in intractable epilepsy. ${ }^{3,11,13,21}$ These studies addressed surgical management strategies, such as the decision to perform surgery versus no surgery and the determination of whether additional electrodes were needed, but did not address in detail other variables, such as the number of electrodes used, removal of unnecessary electrodes, and subdural electrode repositioning to cover areas of interest. While the clinical impact of some of these changes is hard to estimate, it is not negligible. The use of larger numbers of electrodes is associated with increased chances of complications, and placement of electrodes in certain areas, such as interhemispheric strips, is not without risks. ${ }^{17,28}$ In the patient cohort of the present study, MSI results allowed for reduction of the number of electrodes in 9 patients (16\%), with the most common reason being removal of unnecessary interhemispheric strips, as MSI provided a better localization hypothesis. MSI results guided reposition- ing of a subdural grid array in another $6(11 \%)$ patients. MSI resulted in increased numbers of contacts in 5 patients. In 3 of these patients, seizures were captured during icEEG arising from MSI electrodes, and no seizures were captured during icEEG in 1 patient.

In our cohort, MSI was instrumental in the surgical decision-making for 12 patients $(21 \%)$ through the detection of subtle cortical malformations or guiding more dedicated sampling to areas often unexplored during invasive monitoring, such as the insula, orbitofrontal region, or precuneus. These findings are in line with those from other prospective studies of patients who are typically considered for icEEG (Table 2). Knowlton et al..$^{13}$ found that MSI results in modified electrode coverage in $23 \%$ of icEEG cases. In $39 \%$ of cases, seizure onset icEEG patterns involved the additional electrodes indicated by MSI. In another study of 69 patients with refractory nonlesional focal epilepsy, MSI results led to changes in surgical treatment decisions for $33 \%$ of patients and were clearly beneficial in $9 \%$ of patients who underwent resection. ${ }^{21}$ Both studies included lesional patients unless it was thought that lesionectomy alone would be sufficient, i.e., cases with a static lesion with no conflicting data ${ }^{21}$ or contrast enhancement suggestive of a neoplastic process. ${ }^{11,13}$ In our cohort, we included only patients with nonlesional epilepsy, as the presence of a lesion will likely influence surgical decisionmaking and electrode placement. Two other prospective studies evaluated patients with less restrictive inclusion criteria, including all patients who underwent presurgical evaluation. De Tiège et al. found that MSI led to changes in the management plan in $21 \%$ of all patients and $28 \%$ of patients with abnormal MEG findings. ${ }^{3}$ MSI was less beneficial in patients with temporal than in those with extratem- 
TABLE 2. Prospective MEG studies addressing the role of MSI in epilepsy surgery

\begin{tabular}{|c|c|c|c|c|}
\hline Authors \& Year & $\begin{array}{l}\text { No. of } \\
\text { Pts }\end{array}$ & Rationale & Outcome & Exclusion Criteria \\
\hline $\begin{array}{l}\text { Knowlton et al., } \\
2006\end{array}$ & 49 & $\begin{array}{l}\text { Examine agreement btwn } \\
\text { MSI \& icEEG localiza- } \\
\text { tion in epilepsy op } \\
\text { candidates }\end{array}$ & $\begin{array}{l}55 \% \text { localized \& concordant for site, } 6 \% \\
\text { localized w/ both tests but discordant for } \\
\text { site, } 14 \% \text { localized w/ icEEG but not w/ } \\
\text { MSI, } 6 \% \text { localized w/ MSI but not w/ icEEG }\end{array}$ & $\begin{array}{l}\text { 1) Evidence of unilateral hippocampal sclerosis } \\
\text { on MRI w/ concordant ictal \& interictal EEG } \\
\& \text { consistent Sz semiology; 2) MRI lesions } \\
\text { w/ contrast enhancement or other features } \\
\text { suggesting high-grade neoplasm }\end{array}$ \\
\hline $\begin{array}{l}\text { Sutherling et al., } \\
2008\end{array}$ & 69 & $\begin{array}{l}\text { Effect on epilepsy op } \\
\text { decision-making }\end{array}$ & $\begin{array}{l}\text { MSI changed surgical decision in } 33 \% \text { of pts; } \\
\text { clearly beneficial in } 9 \% \text { of all pts \& } 21 \% \text { of } \\
\text { resection pts }\end{array}$ & $\begin{array}{l}\text { 1) Meets criteria for standard anterior temporal } \\
\text { lobectomy; 2) pts w/ static lesion }>1.0 \mathrm{~cm} \text { w/ } \\
\text { lateralizing EEG \& no conflicting data }\end{array}$ \\
\hline $\begin{array}{l}\text { Knowlton et al., } \\
2009\end{array}$ & 77 & $\begin{array}{l}\text { Effect on electrode place- } \\
\text { ment }\end{array}$ & $\begin{array}{l}\text { Modified electrode coverage in 18/77 (23\%) } \\
\text { icEEG cases; in 39\% Sz onset icEEG } \\
\text { patterns involved additional electrodes } \\
\text { indicated by MSI }\end{array}$ & $\begin{array}{l}\text { 1) Clinically defined mesial temporal lobe epi- } \\
\text { lepsy supported by localized scalp VEEG (in- } \\
\text { cluding supportive semiology) \& concordant } \\
\text { evidence of unilateral hippocampal sclerosis } \\
\text { on MRI; 2) pts considered to require simple } \\
\text { lesionectomy }\end{array}$ \\
\hline $\begin{array}{l}\text { De Tiège et al., } \\
2012\end{array}$ & 70 & $\begin{array}{l}\text { Influence on epilepsy pt } \\
\text { management }\end{array}$ & $\begin{array}{l}\text { MSI changed management plan in } 21 \% \text { of all } \\
\text { pts } \& 28 \% \text { of pts w/ abnormal MEG }\end{array}$ & $\begin{array}{l}\text { Pts formally excluded from op (i.e., multifocal } \\
\text { epilepsy or severe epileptic encephalopathy) }\end{array}$ \\
\hline $\begin{array}{l}\text { Duez et al., } \\
\quad 2019\end{array}$ & 141 & $\begin{array}{l}\text { Diagnostic accuracy \& } \\
\text { clinical utility of EMSI }\end{array}$ & $\begin{array}{l}\text { Changed Tx management plan in } 34 \% \text { of pts; } \\
\text { these changes were useful in } 80 \% \text {; EMSI } \\
\text { had concordance of } 53 \%-89 \% \text { w/ irritative } \\
\text { zone \& } 35 \%-73 \% \text { w } \text { Sz onset zone }\end{array}$ & None; included all pts evaluated for epilepsy op \\
\hline
\end{tabular}

poral epilepsy. In another study analyzing electromagnetic source imaging (EMSI) through 2 different software packages, EMSI findings led to changes in the management plans in $34 \%$ of the patients, and these changes were useful in $80 \%$. EMSI had a concordance of $53 \%-89 \%$ with the irritative zone and $35 \%-73 \%$ with the seizure onset zone. ${ }^{4}$ The above studies and others ${ }^{7,15,18,20}$ collectively point to the role of MSI in the presurgical workup of patients with intractable epilepsy. MSI is likely to impact outcome in approximately $20 \%$ of patients when used as an investigation added to other presurgical assessment modalities.

One additional benefit of MSI is that it allows the detection of subtle imaging abnormalities. MSI-guided review can lead to the detection of previously unidentified lesions, and MSI can be used to enhance the interpretation of MRI data through directed attention to specific brain regions. ${ }^{9}$ Several advanced MRI postprocessing strategies have been studied to improve the detection of subtle cortical abnormalities in the EZ that might not be detected through conventional visual interpretation. Among these, a voxel-based MRI morphometric analysis program (MAP) has been used to detect subtle cortical malformations. ${ }^{25}$ We believe that both MRI postprocessing and MSI are complementary techniques that can provide valuable information in patients with NLRFE, and the superiority of one over the other can only be demonstrated through prospective randomized trials assessing the utility of both techniques. The finding of a concordant MSI/MAP-positive area can become an important determinant of an epileptogenic lesion and can facilitate hypothesis-driven, optimal placement of a relatively small number of implanted electrodes. ${ }^{24}$

\section{Study Limitations}

It is important to point out some of the limitations of this study. While MSI results affected patient management, the actual impact on surgical outcome is difficult to assess. We previously demonstrated that MSI could change surgical outcomes in a unique patient population for which MEG data were acquired prior to surgery but not used clinically, resulting in surgical failures in some cases. ${ }^{14}$

This study reflects the practice in a single epilepsy referral center. We used subdural electrodes to map the EZ, with additional depth electrodes when further invasive monitoring was needed. Subdural electrodes are relatively insensitive to deep epileptic foci, and exploration is often limited to one hemisphere. Patients with a normal MRI and electroclinical features indicative of involvement in a functional network may require sampling from several brain regions. In those patients, stereo-electroencephalography (SEEG) may be more suitable for invasive evaluation. ${ }^{10,23}$ In a retrospective series of 50 patients who underwent SEEG and MSI, adequate sampling of the MEG cluster area with SEEG electrodes carried a higher chance for a seizure-free outcome. ${ }^{15}$ Complete resection of the MEG cluster was associated with a seizure-free outcome, but complete resection of the ictal onset areas based on SEEG alone was not. The value of MSI in patients undergoing SEEG implantation might even be higher because of the inherently sparse sampling of SEEG electrodes, and prospective evaluation of the utility of MSI in patients undergoing invasive monitoring using SEEG electrodes might further validate this observation.

Some of the patients in this cohort were excluded from surgery because of overlap of an MSI cluster with eloquent 
cortex areas, and hence the validity of MSI results in relation to surgical outcomes in these patients cannot be established. The increasing availability of responsive neurostimulation devices might allow some of those patients to be surgical candidates.

\section{Conclusions}

This prospective, blinded study showed that MSI can affect icEEG planning and surgical strategy in patients with NLRFE, and it can potentially affect outcomes in $21 \%$ of patients with NLRFE. The prospective utility of MSI in patients undergoing invasive monitoring exclusively using SEEG could be even higher and should be studied separately.

\section{Acknowledgments}

Dr. Nguyen received funding from the CHUM Foundation (93512), FRQS clinical research scholar program (31040), and Canada Research Chair (950-232075), and a CIHR project grant (390044). Dr. Mohamed received funding from the NSF (1632891).

\section{References}

1. Assaf BA, Karkar KM, Laxer KD, Garcia PA, Austin EJ, Barbaro NM, et al: Magnetoencephalography source localization and surgical outcome in temporal lobe epilepsy. Clin Neurophysiol 115:2066-2076, 2004

2. Berg AT, Vickrey BG, Langfitt JT, Sperling MR, Walczak TS, Shinnar S, et al: The multicenter study of epilepsy surgery: recruitment and selection for surgery. Epilepsia 44:1425-1433, 2003

3. De Tiège X, Carrette E, Legros B, Vonck K, Op de Beeck $\mathrm{M}$, Bourguignon $\mathrm{M}$, et al: Clinical added value of magnetic source imaging in the presurgical evaluation of refractory focal epilepsy. J Neurol Neurosurg Psychiatry 83:417-423, 2012

4. Duez L, Tankisi H, Hansen PO, Sidenius P, Sabers A, Pinborg LH, et al: Electromagnetic source imaging in presurgical workup of patients with epilepsy: a prospective study. Neurology 92:e576-e586, 2019

5. Ebersole JS, Ebersole SM: Combining MEG and EEG source modeling in epilepsy evaluations. J Clin Neurophysiol 27:360-371, 2010

6. Engel J Jr, Van Ness PC, Rasmussen TB, Ojemann LM: Outcome with respect to epileptic seizures, in Engel J Jr (ed): Surgical Treatment of the Epilepsies, ed 2. New York: Raven Press, 1993, pp 609-621

7. Englot DJ, Nagarajan SS, Imber BS, Raygor KP, Honma SM, Mizuiri D, et al: Epileptogenic zone localization using magnetoencephalography predicts seizure freedom in epilepsy surgery. Epilepsia 56:949-958, 2015

8. Fujimoto A, Ochi A, Imai K, Chan D, Sharma R, Viljoen A, et al: Magnetoencephalography using total intravenous anesthesia in pediatric patients with intractable epilepsy: lesional vs nonlesional epilepsy. Brain Dev 31:34-41, 2009

9. Funke ME, Moore K, Orrison WW Jr, Lewine JD: The role of magnetoencephalography in "nonlesional" epilepsy. Epilepsia 52 (Suppl 4):10-14, 2011

10. Gonzalez-Martinez J, Bulacio J, Alexopoulos A, Jehi L, Bingaman W, Najm I: Stereoelectroencephalography in the "difficult to localize" refractory focal epilepsy: early experience from a North American epilepsy center. Epilepsia 54:323-330, 2013

11. Knowlton RC, Elgavish R, Howell J, Blount J, Burneo JG, Faught E, et al: Magnetic source imaging versus intracranial electroencephalogram in epilepsy surgery: a prospective study. Ann Neurol 59:835-842, 2006

12. Knowlton RC, Elgavish RA, Limdi N, Bartolucci A, Ojha $\mathrm{B}$, Blount J, et al: Functional imaging: I. Relative predictive value of intracranial electroencephalography. Ann Neurol 64:25-34, 2008

13. Knowlton RC, Razdan SN, Limdi N, Elgavish RA, Killen J, Blount J, et al: Effect of epilepsy magnetic source imaging on intracranial electrode placement. Ann Neurol 65:716-723, 2009

14. Mohamed IS, Bouthillier A, Bérubé A, Cossette P, Finet P, Saint-Hilaire JM et al: The clinical impact of integration of magnetoencephalography in the presurgical workup for refractory nonlesional epilepsy. Epilepsy Behav 79:34-41, 2018

15. Murakami H, Wang ZI, Marashly A, Krishnan B, Prayson RA, Kakisaka Y, et al: Correlating magnetoencephalography to stereo-electroencephalography in patients undergoing epilepsy surgery. Brain 139:2935-2947, 2016

16. Oishi M, Kameyama S, Masuda H, Tohyama J, Kanazawa O, Sasagawa M, et al: Single and multiple clusters of magnetoencephalographic dipoles in neocortical epilepsy: significance in characterizing the epileptogenic zone. Epilepsia 47:355-364, 2006

17. Onal C, Otsubo H, Araki T, Chitoku S, Ochi A, Weiss S, et al: Complications of invasive subdural grid monitoring in children with epilepsy. J Neurosurg 98:1017-1026, 2003

18. Rampp S, Stefan H, Wu X, Kaltenhäuser M, Maess B, Schmitt FC, et al: Magnetoencephalography for epileptic focus localization in a series of 1000 cases. Brain 142:30593071, 2019

19. Rosenow F, Lüders H: Presurgical evaluation of epilepsy. Brain 124:1683-1700, 2001

20. Stefan H, Hummel C, Scheler G, Genow A, Druschky K, Tilz $\mathrm{C}$, et al: Magnetic brain source imaging of focal epileptic activity: a synopsis of 455 cases. Brain 126:2396-2405, 2003

21. Sutherling WW, Mamelak AN, Thyerlei D, Maleeva T, Minazad Y, Philpott L, et al: Influence of magnetic source imaging for planning intracranial EEG in epilepsy. Neurology 71:990-996, 2008

22. Téllez-Zenteno JF, Hernández Ronquillo L, Moien-Afshari F, Wiebe S: Surgical outcomes in lesional and non-lesional epilepsy: a systematic review and meta-analysis. Epilepsy Res 89:310-318, 2010

23. Vadera S, Mullin J, Bulacio J, Najm I, Bingaman W, Gonzalez-Martinez J: Stereoelectroencephalography following subdural grid placement for difficult to localize epilepsy. Neurosurgery 72:723-729, 2013

24. Wang ZI, Alexopoulos AV, Jones SE, Najm IM, Ristic A, Wong C, et al: Linking MRI postprocessing with magnetic source imaging in MRI-negative epilepsy. Ann Neurol 75:759-770, 2014

25. Wellmer J, Parpaley Y, von Lehe M, Huppertz HJ: Integrating magnetic resonance imaging postprocessing results into neuronavigation for electrode implantation and resection of subtle focal cortical dysplasia in previously cryptogenic epilepsy. Neurosurgery 66:187-195, 2010

26. Wheless JW, Castillo E, Maggio V, Kim HL, Breier JI, Simos PG, et al: Magnetoencephalography (MEG) and magnetic source imaging (MSI). Neurologist 10:138-153, 2004

27. Wiebe S, Jetté N: Epilepsy surgery utilization: who, when, where, and why? Curr Opin Neurol 25:187-193, 2012

28. Wong CH, Birkett J, Byth K, Dexter M, Somerville E, Gill $\mathrm{D}$, et al: Risk factors for complications during intracranial electrode recording in presurgical evaluation of drug resistant partial epilepsy. Acta Neurochir (Wien) 151:37-50, 2009

\section{Disclosures}

The authors report no conflict of interest concerning the materi- 
als or methods used in this study or the findings specified in this paper.

\section{Author Contributions}

Conception and design: Nguyen, Mohamed, Bouthillier. Acquisition of data: Nguyen, Toffa, Robert, Cossette, Bérubé, Saint-Hilaire, Bouthillier. Analysis and interpretation of data: all authors. Drafting the article: Nguyen, Mohamed, Toffa. Critically revising the article: Nguyen, Mohamed, Toffa. Reviewed submitted version of manuscript: all authors. Approved the final version of the manuscript on behalf of all authors: Nguyen. Statistical analysis: Mohamed. Administrative/technical/material support: Nguyen, Toffa. Study supervision: Nguyen, Mohamed.

\section{Supplemental Information}

Previous Presentations

The concept, design, and preliminary results of this work were part of a PhD thesis (D.K.N.): Nguyen DK: Apport de nouvelles techniques dans l'évaluation de patients candidats à une chirurgie d'épilepsie: résonance magnétique à haut champ, spectroscopie proche infrarouge et magnétoencéphalographie [PhD dissertation]. Montreal, QC, Canada: Université de Montréal, 2013. Retrieved from the Papyrus database. (http://hdl.handle. net/1866/10910).

\section{Correspondence}

Dang Khoa Nguyen: Montreal University Health Center, Montreal, QC, Canada.d.nguyen@umontreal.ca. 